- Letter

\title{
Validity of Alcohol Use Disorder Identification Test: Methodological Issues
}

\author{
Siamak Sabour ${ }^{1,2, *}$ \\ 'Safety Promotion and Injury Prevention Research Center, Shahid Beheshti University of Medical Sciences, Tehran, Iran \\ ${ }^{2}$ Department of Clinical Epidemiology, Shahid Beheshti University of Medical Sciences, Tehran, Iran
}

\section{To the Editor}

I was interested to read the paper by Chang et al. ${ }^{1)}$ that was published in the November 2016 edition of the Korean Journal of Family Medicine. The purpose of the authors was to evaluate the validity of the Alcohol Use Disorder Identification TestKorean revised version (AUDIT-KR) for screening alcohol use disorders (AUDs) in accordance with the Diagnostic and Statistical Manual of Mental Disorders, fifth edition (DSM-5) criteria. ${ }^{1)}$ The study included 443 subjects who completed the AUDIT-KR. The subjects were divided into two groups, an AUD group and a non-AUD group, according to the DSM-5 criteria. Based on their report, the optimal cutoff score in the AUDIT-KR was 10 points for males (sensitivity, $81.9 \%$; specificity, $81.3 \%$; positive predictive value, $77.2 \%$; and negative predictive value, $85.3 \%$ ) and 5 points for females (sensitivity, $100.0 \%$; specificity, $88.5 \%$; positive predictive value, $52.6 \%$; and negative predictive value, $100.0 \%) .^{1)}$

Reported estimates are usually used to assess the validity of a single test instead of a questionnaire. To validate the revised version of any test, the suggestion is to focus on face and content validity, and probably on structure validity. However, considering the limitations of the mentioned estimates (positive predictive value depends on prevalence), why did the authors not use positive and negative likelihood ratios (LR+ and LR-), diagnostic accuracy, and odds ratio (ratio of true results to false results)? ${ }^{2-9)}$ Finally, when the author reported area under the receiver-operating characteristic curve of the AUDITKR for identifying AUD, they actually reported the validity (ac- curacy and discrimination) of the model instead of the test. Therefore, it is crucial to not confuse a single test with a questionnaire or a diagnostic model because assessment of the validity of the mentioned concepts is completely different. ${ }^{2-9)}$

They concluded that the AUDIT-KR has high reliability and validity for identifying AUD in accordance with the DSM- 5 criteria. Reliability (precision and calibration) is a different methodological issue from validity (accuracy and discrimination). ${ }^{2)}$ Therefore, such conclusion may be misleading because of the inappropriate use of statistical tests to assess validity and confusing the concept of validity and reliability, and not differentiating between a single test with a questionnaire or diagnostic model.

\section{CONFLICT OF INTEREST}

No potential conflict of interest relevant to this article was reported.

\section{REFERENCES}

1. Chang JW, Kim JS, Jung JG, Kim SS, Yoon SJ, Jang HS. Validity of Alcohol Use Disorder Identification Test-Korean revised version for screening alcohol use disorder according to Diagnostic and Statistical Manual of Mental Disorders, fifth edition criteria. Korean J Fam Med 2016;37:323-8.

2. Szklo M, Nieto FJ. Epidemiology beyond the basics. 2nd ed. Sudbury (MA): Jones and Bartlett Publishers; 2007.

3. Sabour S. Reliability of a new modified tear breakup time method: methodological and statistical issues. Graefes Arch 
Clin Exp Ophthalmol 2016;254:595-6.

4. Sabour S, Ghassemi F. The validity and reliability of a signal impact assessment tool: statistical issue to avoid misinterpretation. Pharmacoepidemiol Drug Saf 2016;25:1215-6.

5. Sabour S. Adherence to guidelines strongly improves reproducibility of brachial artery flow-mediated dilation: common mistakes and methodological issue. Atherosclerosis 2016;251:490-1.

6. Sabour S, Farzaneh F, Peymani P. Evaluation of the sensitivity and reliability of primary rainbow trout hepatocyte vitellogenin expression as a screening assay for estrogen mimics: methodological issues. Aquat
Toxicol 2015;164:175-6.

7. Sabour S. Reliability of the ASA physical status scale in clinical practice: methodological issues. Br J Anaesth 2015;114:162-3.

8. Sabour S, Ghassemi F. Pediatric FOUR Score Coma Scale: interrater reliability and predictive validity-mistake and misinterpretation. J Neurosci Nurs 2014;46:369-70.

9. Sabour S. Accuracy and reproducibility of dental measurements using different technologies, methodologic mistake. Am J Orthod Dentofacial Orthop 2014;145:549-50. 